\title{
Saúde e qualidade de vida dos agentes de nível médio do trabalho de enfermagem a
}

\section{partir da terceira idade}

\author{
Health and quality of life of mid-level nursing workers from the elderly \\ Salud y calidad de vida de trabajadores de enfermería de nivel medio de ancianos
}

Recebido: 18/10/2021 | Revisado: 25/10/2021 | Aceito: 29/10/2021 | Publicado: 01/11/2021

\author{
João Pedro Neto de Sousa \\ ORCID: https://orcid.org/0000-0001-5974-3544 \\ Universidade Federal de Mato Grosso, Brasi \\ E-mail: jpneto_@live.com \\ Antônio César Ribeiro \\ ORCID: https://orcid.org/0000-0003-1607-3215 \\ Universidade Federal de Mato Grosso, Brasil \\ E-mail: anceri1964@gmail.com \\ Juliano Bortolini \\ ORCID: https://orcid.org/0000-0003-0126-3040 \\ Universidade Federal de Mato Grosso, Brasil \\ E-mail: julianobortolini@ufmt.br
}

\begin{abstract}
Resumo
A investigação da qualidade de vida relacionado a saúde profissional é relevante podendo esta refletir na saúde do profissional de enfermagem e assim impactar em sua qualidade de vida relacionada à saúde. Objetivo: analisar o perfil de saúde e a qualidade de vida relacionada à saúde dos agentes de nível médio da enfermagem a partir da terceira idade. Método: Estudo transversal de abordagem quantitativa, com os agentes de nível médio da enfermagem na terceira idade, com aplicação de roteiro norteador além do instrumento The Medical Outcomes Study 36-item ShortForm Health Survey (SF-36), durante o segundo semestre de 2019. Os dados coletados foram organizados e tabulados por meio da frequência absoluta (n) e relativa (\%) e apresentados em tabelas, além da realização do teste ShapiroWilk para verificar a normalidade dos dados e após procedeu-se com teste não paramétrico para medianas de KruskalWallis, que quando significativo $(\mathrm{p}<0,05)$, realizou-se o teste de Conover-Iman para a comparação múltipla entre as medianas. Resultados: fatores como obesidade, sedentarismo, limitações físicas, doenças crônicas e ocupacionais, sobrecarga e desgastes oriundos do trabalho, foram identificados como agravantes à saúde e qualidade de vida dos profissionais. Com aplicação do instrumento SF-36 observou-se que o domínio aspectos sociais apresentou melhor escore médio, enquanto o domínio de estado geral da saúde refletiu um escore de menor média. Conclusão: Ademais, conclui-se que a enfermagem ainda carece de melhores condições em suas atividades laborais e de novas estratégias para o enfrentamento do adoecimento na enfermagem.
\end{abstract}

Palavras-chave: Qualidade de vida; Enfermagem; Terceira idade; Saúde.

\begin{abstract}
The investigation of quality of life related to professional health becomes relevant and may reflect on the health of the nursing professional and thus impact on their health-related quality of life. Objective: to analyze the health profile and health-related quality of life of mid-level nursing agents from old age onwards. Method: Cross-sectional study with a quantitative approach, with mid-level nursing agents in the third age, with application of a semi-structured script in addition to the instrument The Medical Outcomes Study 36-item Short-Form Health Survey (SF-36), during the second semester of 2019. The collected data were organized and tabulated using the absolute (n) and relative (\%) frequency and presented in tables, in addition to the Shapiro-Wilk test to verify the normality of the data and then proceeded with the non-test. parametric for Kruskal-Wallis medians, which when significant $(\mathrm{p}<0.05)$, the ConoverIman test was used for multiple comparisons between the medians. Results: factors such as obesity, sedentary lifestyle, physical limitations, chronic and occupational diseases, overload and strain from work were identified as aggravating the health and quality of life of professionals. With the application of the SF-36 instrument, it was observed that the social aspects domain had a better mean score, and the general health domain had a lower mean score. Conclusion: Furthermore, it is concluded that nursing still lacks better conditions in their work activities and new strategies for coping with illness in nursing.
\end{abstract}

Keywords: Quality of life; Nursing; Elderly. 


\begin{abstract}
Resumen
La investigación de la calidad de vida relacionada con la salud profesional cobra relevancia y puede reflejarse en la salud del profesional de enfermería y por lo tanto impactar en su calidad de vida relacionada con la salud. Objetivo: analizar el perfil de salud y la calidad de vida relacionada con la salud de los agentes de enfermería de nivel medio a partir de la vejez. Método: Estudio transversal con abordaje cuantitativo, con agentes de enfermería de nivel medio en la tercera edad, con aplicación de un guión semiestructurado además del instrumento The Medical Outcomes Study 36-ítems Short-Form Health Survey (SF- 36), durante el segundo semestre de 2019. Los datos recolectados fueron organizados y tabulados utilizando la frecuencia absoluta (n) y relativa (\%) y presentados en tablas, además de la prueba de Shapiro-Wilk para verificar la normalidad de los datos y luego se procedió con la no prueba paramétrica para medianas de Kruskal-Wallis, que cuando fue significativa ( $\mathrm{p}<0.05$ ), se utilizó la prueba de Conover-Iman para comparaciones múltiples entre las medianas. Resultados: se identificaron factores como la obesidad, el sedentarismo, las limitaciones físicas, las enfermedades crónicas y ocupacionales, la sobrecarga y el esfuerzo del trabajo como agravantes de la salud y la calidad de vida de los profesionales. Con la aplicación del instrumento SF-36, se observó que el dominio de aspectos sociales presentó una mejor puntuación media y el dominio de salud general una menor puntuación media. Conclusión: Además, se concluye que la enfermería aún carece de mejores condiciones en sus actividades laborales y nuevas estrategias para el afrontamiento de la enfermedad en enfermería.
\end{abstract}

Palabras clave: Calidad de vida; Enfermería; Tercera edad.

\title{
1. Introdução
}

A enfermagem, como parte do trabalho em saúde, tem sua institucionalidade assentada em normas legais, que a definem como uma "profissão" estratificada e fortemente marcada pela divisão técnica ou pormenorizada do trabalho, que é executado por diferentes agentes. Neste sentido, estão a cargo dos agentes de nível médio, o auxiliar de enfermagem e o técnico de enfermagem, a assunção de atividades de natureza simples e repetitiva com o predomínio do esforço físico (Brasil, $1986 ; 1987)$.

Dentre as características peculiares a esse grupo de trabalhadores podem ser citadas: o ambiente laboral insalubre e estressante; a relação com o objeto de trabalho - o corpo humano sadio ou doente, por vezes em sofrimento e/ou situação de morte; as jornadas extensas de trabalho, inclusive noturnas; os baixos salários e a baixa valorização, dada a perspectiva de secundarização da prática da enfermagem pelas organizações; os conflitos organizacionais decorrentes da forte hierarquia e verticalização de comando, como características históricas da forma de organização do trabalho da enfermagem; o multiemprego, decorrente da baixa remuneração e necessidades de garantia das condições materiais de existência dos seus agentes (Ribeiro, Souza, Silva, 2014).

Tais condições de trabalho muitas vezes a que estão expostos essa classe profissional acarreta desgastes a estes trabalhadores, sobretudo para seus agentes de nível médio, nos quais os efeitos deletérios do trabalho são mais intensos, fato atribuído à natureza das suas ações supracitadas e das condições vividas durante suas atividades, o que reflete em sua saúde e Qualidade de Vida (QV) (Amaral; Ribeiro; Paixão, 2015; Tanferri et al., 2017).

Com relação a isto, Tanferri et al. (2017), pressupõem em seu estudo que as especificidades existentes no processo laboral dos agentes de nível médio da enfermagem, tais como a sobrecarga e as condições de trabalho, são fatores que também implicaram na relação com o tempo de serviço na enfermagem, uma vez que quanto maior o tempo de trabalho desses agentes, menor foi a QV na aposentadoria.

Ao correlacionar esses fatores ocasionados no trabalho destes profissionais com o processo de envelhecimento, as cargas de trabalho permanecem e frequentemente aumentam, entretanto, a capacidade de pessoas mais idosas suportar essa rotina de trabalho mais intensa, muitas vezes, é reduzida. Diante disso, é fato que as condições de trabalho e o envelhecimento, correlacionados à idade e ao tempo de serviço dos profissionais de enfermagem, apresentam uma associação significativa, uma vez que, com o passar da idade, ocorre uma perda da capacidade física para a execução do trabalho (Pires et al., 2013).

Diante ao exposto, enaltece-se a preocupação com a qualidade de vida dos agentes de nível médio da enfermagem, principalmente os acima de 60 anos de idade, estando em atividade e/ou aposentados, visto que esta é uma fase de diversas 
alterações fisiológicas e psicossociais que necessitam de acompanhamento, não somente pelo próprio indivíduo, mas pelo mundo do trabalho, uma vez que as suas condições de saúde estão na dependência direta das condições laborais durante a sua vida produtiva (Freire; Costa, 2016).

Embora haja estudos relacionados à aposentadoria e à terceira idade de uma forma geral, esta pesquisa aborda uma lacuna existente e específica: como é o perfil de saúde e a qualidade de vida relacionada à saúde entre os agentes de nível médio da enfermagem na terceira idade? A fim de identificar e explorar de forma mais ampliada a situação em que esses profissionais se encontram, pretende-se, ainda, despertar sobre a promoção e prevenção de agravos à saúde dos trabalhadores em seu ambiente de trabalho e os impactos que este pode oferecer para a sua vida.

\section{Metodologia}

Trata-se de um estudo epidemiológico com delineamento transversal, descritivo e exploratório. O estudo epidemiológico é aquele que possui características de avalição da distribuição de doenças e sobre a saúde da população em uma determinada área de escolha. Este pode assumir o delineamento transversal, que segundo o mesmo autor é o tipo de estudo cujas relações de causa e efeito são analisadas ao mesmo tempo, caracterizando uma observação direta em uma única oportunidade (Medronho, 2009).

O estudo de corte transversal é assinalado pela observação direta de um evento com uma quantidade determinada de indivíduos em uma única oportunidade (Medronho, 2009). A pesquisa descritiva objetiva apresentar características de determinada população ou fenômenos, utilizando técnicas padronizadas de coleta de dados (Gil,2010). Propõe a observação, registro, análise, classificação e interpretação dos dados sem a interferência do pesquisador (Andrade, 2002). A pesquisa é ainda de caráter exploratório dado o contexto incipiente de investigações sobre o tema, sendo este tipo de pesquisa realizada no intuito de identificar uma visão geral sobre determinado objeto. É realizada quando o tema é pouco explorado e de difícil formulação de hipóteses. Portanto busca-se conhecer com maior profundidade o assunto para que se torne possível construir questões importantes para o desenvolvimento do estudo (Gil,2010).

A pesquisa foi realizada no município de Cuiabá- MT, sendo este escolhido em razão da concentração populacional, no qual o município apresenta um alto contingente de profissionais de nível médio da enfermagem acima da terceira idade quando comparado a outros municípios do Estado, segundo informações colhidas no Setor de Registro e Cadastro do CorenMT, mediante autorização expressa do Conselho Regional de Enfermagem do Estado de Mato Grosso (COREn - MT), que forneceu os dados relativos ao contato dos indivíduos que foram utilizados, exclusivamente, para fins da realização do presente estudo e, posteriormente incinerados.

O estudo teve como participantes profissionais de nível médio da área de enfermagem (técnicos e auxiliares de enfermagem). Os indivíduos de interesse para a presente investigação, segundo as informações do Setor de Registro e Cadastro do Coren-MT, foram 933 indivíduos, entretanto, para o cálculo do tamanho da amostra para aplicação dos questionários, considerou-se: população de $\mathrm{N}=933$, que corresponde aos profissionais de nível médio da enfermagem, excluindo os profissionais com dupla inscrição no COREN (técnico e auxiliar de enfermagem). Assim sendo, a população final resultou em $\mathrm{N}=810$ indivíduos, independente da sua situação cadastral (ativo, ativo/remido ou cancelado), com o intervalo de confiança de 95\% $\left(z_{\alpha / 2}=1,96\right)$, uma proporção de 50\% para prevalência desconhecida e erro amostral de 5\%.

Por meio dos referidos cálculos, definiu-se uma amostra mínima de 287 profissionais, já inclusos $10 \%$ no valor final por possíveis perdas/recusas, onde optou-se pela amostra estratificada simples, na qual os indivíduos foram sorteados indistintamente até o número total da amostra. 
Para seleção foram considerados como critérios de inclusão: se profissional de nível médio de enfermagem, estando em atividade laboral ou aposentado por idade ou por invalidez, de ambos os sexos; residir em Cuiabá; ter completado 60 anos até 31 de dezembro de 2018, e aceitar participar da pesquisa assinando o Termo de Consentimento Livre Esclarecido (TCLE).

O trabalho de campo ocorreu de julho a novembro do ano de 2019 com auxílio de um roteiro norteador elaborado pelos pesquisadores por meio de revisões para sua construção, e o instrumento The Medical Outcomes Study 36-item ShortForm Health Survey (SF-36), traduzido e validado para o Brasil em 1997, que foi orientado para o autopreenchimento.

O questionário estruturado que contemplou as seguintes variáveis independentes: Informações sociodemográficas (idade, local de nascimento: UF, sexo, estado civil, filhos, chefe da família), Critério de Classificação Econômica Brasil ABEP - Associação Brasileira de Empresas de Pesquisa (ABEP, 2019) (Itens de consumo em sua casa categorizados pela quantidade, grau de instrução do chefe de família, acesso a serviços públicos, plano de saúde), História profissional (Situação profissional atual, maior escolaridade, vínculo de maior permanência no trabalho, ano de ingresso no primeiro emprego, carga horária semanal mais praticada; área de atuação de maior permanência, regime de trabalho mais praticado, turno de trabalho, possui ou já possui mais de um vínculo), Hábitos e perfil de saúde ( Tabagista, etilismo, automedicação, agravo/doença crônica, uso regular de medicamento, história de doença ocupacional, limitação física para o trabalho, portador de necessidades especiais, familiar dependente de seus cuidados por agravo à saúde, peso e estatura (autorreferidas), prática atividade física, lazer.

Como variável dependente foi aplicado o instrumento Qualidade de vida (SF-36), que é um questionário genérico, do tipo Likert, multidimensional, constituído por 36 itens, englobados em 8 escalas ou componentes: capacidade funcional (10 itens), aspectos físicos (4 itens), dor (2 itens), estado geral de saúde (5 itens), vitalidade (4 itens), aspectos sociais (2 itens), aspectos emocionais ( 3 itens), saúde mental (5 itens) e mais uma questão de avaliação comparativa entre as condições de saúde atual e a de um ano atrás. Esta tem como objetivo avaliar tantos os aspectos negativos de uma determinada condição enferma como também os pontos positivos de bem-estar, em uma escala que varia de 0 a 100 (obtido por meio de cálculo do RawScale), na qual 0 corresponde ao pior estado geral de saúde e 100 ao melhor estado de saúde ( Ribeiro et al., 2018).

A identificação dos instrumentos foi processada mediante a atribuição, em campo específico, do código composto pelas iniciais maiúsculas do nome do indivíduo, seguidas pela ordem dos números escolhidos pelo pesquisador, tendo o primeiro envelope recebido o número de 10001 e o último de 10287, por exemplo, João Pedro Neto de Sousa, Código 10001 (JPNS10001).

$\mathrm{Na}$ análise de dados, estes foram duplamente digitados como forma de monitorar possíveis inconsistências ou erros no programa Epi info versão 3.1. Após, foram organizadas e realizadas as análises descritivas, considerando a distribuição e frequência dos dados, dispondo-os em tabelas.

Para as demais análises, utilizou-se o Software R (R Core Team, 2019), o qual realizou o teste Shapiro-Wilk para verificar a normalidade dos dados. Após a constatação da não normalidade, procedeu-se com o teste não paramétrico para medianas Kruskal-Wallis. Quando o teste de Kruskal-Wallis foi significativo $(\mathrm{p}<0,05)$, realizou-se o teste de Conover-Iman para a comparação múltipla entre as medianas.

Esta pesquisa parte de um projeto matricial intitulado "Saúde e qualidade de vida dos trabalhadores de enfermagem a partir da terceira idade", aprovado pelo Comitê de Ética em Pesquisa em Saúde com Seres Humanos da Universidade Federal de Mato Grosso - Campus Cuiabá, tendo como número de Certificado de Apresentação para Apreciação Ética (CAAE) 89197118.5.0000.812, com número do parecer 2.670.711.

Assim, essa pesquisa respeitou todos os princípios éticos e as normas regulamentadoras de pesquisa com seres humanos em todas as suas fases, conforme prevê a Resolução 466, de 12 de dezembro de 2012, do Conselho Nacional de 
Saúde e Resolução 510, de 07 de abril de 2016, do Conselho Nacional de Saúde (Brasil, 2012, 2016), assim como a resolução 311/2007 do Conselho Federal de Enfermagem.

\section{Resultados e Discussão}

Em relação ao perfil dos participantes do estudo, houve uma predominância do sexo feminino com 96,52\%. Reforçando essa premissa, Wermelinger et al., (2010) e Meira et al., (2017), ao delinear as atividades estruturais no Brasil, principalmente as do setor de serviços de saúde, identificaram uma forte preponderância da força de trabalho feminina, especialmente naquelas que envolvem o trato e o cuidado com as pessoas.

Com relação ao local de nascimento dos participantes, este se concentrou na região Centro-Oeste, com 84,32\%, o que pode ser atribuído a maior visibilidade que a enfermagem ganhou no ultimo tempos, com evoluções desde o final da década de 50 ampliando o campo de trabalho com surgimento de cursos de auxiliares de enfermagem e, posteriormente, a criação do Curso de Graduação em Enfermagem da Universidade Federal de Mato Grosso - CGEUFMT, o que fomentou a profissionalização das pessoas da região (Moreira; Ramos, 2004; Flores; Almeida; Junior, 2017).

Quanto à faixa etária, a maioria dos agentes de nível médio da enfermagem se encontravam na faixa de 60 a 64 anos, o que correspondeu a 35,19\% dos indivíduos. Segundo Machado et al., (2016a), esta é a fase da "aposentadoria", na qual os profissionais já se retiraram totalmente ou parcialmente do mercado de trabalho.

No que concerne ao estado civil, 36,93\% correspondem aos que se declararam casados ou em união estável, identificando neste estudo uma maior prevalência de indivíduos que não possuem um companheiro (a). Sendo as sim, podemos correferir com os 1.389 .823 agentes de nível médio da enfermagem observados no estudo do perfil sociodemográfico da enfermagem brasileira, exposto por Machado et al., (2016a), em que 52\% desses profissionais declararam não possuir um companheiro.

Aqueles que afirmaram ter filhos representam 90,59\%, tendo o número de filhos de 1 a 3 representados por 60,63\%, entretanto o número de filhos entre estes profissionais tem apontado uma recorrente baixa percentual, visto que um estudo observou que 54\% dos profissionais de enfermagem entrevistados tinham 1 ou 2 filhos e $26 \%$ não tinham. Podemos correlacionar este fato ao de que a cada dia a aptidão da mulher para o trabalho fora de casa vem crescendo, e impacta direta e/ou indiretamente nas tarefas exclusivas do ambiente doméstico como por exemplo, tempo para ter filhos (Souza et al., 2012; Santos et al., 2017).

Com base nesse argumento, outro contexto que ressalta o papel das mulheres cada vez mais importante é o de provedora da casa e/ou de chefe de família, em que a maioria dos entrevistados se autodeclararam chefes de família, apresentaram o maior percentual, 72,82\%. Fato esse que vem sendo mostrado em diversos estudos, materializando diversos movimentos de transformação social pós-moderna na vida das mulheres, na qual a chefia feminina se consolida como um avanço das mulheres na sociedade, principalmente no mercado de trabalho, fortalecendo a independência e a emancipação feminina nesse universo (Mendes, 2016; Santos et al., 2017).

A análise dos dados oriundos das respostas revelou um campo amplo ao qual a enfermagem está submetida que acaba a propiciar danos à sua saúde, como apresentados nos subtópicos: histórico profissional dos agentes de nível médio da enfermagem; hábitos de vida e perfil de saúde e qualidade de vida relacionada à saúde.

Após, é também apresentado a diferenciação estatística entre as variáveis dependente e independente sendo para este utilizado o teste de Kruskal-Wallis. 


\subsection{Histórico profissional dos agentes de nível médio da enfermagem}

Quanto à formação, é possível destacar que $65,51 \%$ dos participantes afirmaram possuir ensino médio completo, o que corrobora com outros estudos sobre a exigência mínima para a formação tanto do auxiliar quanto do técnico de enfermagem. Entretanto, vale destacar que cerca de $22 \%$ dos indivíduos entrevistados estão cursando ou já obtêm nível superior, o que sinaliza para o fenômeno da superqualificação dos trabalhadores, que desde os anos 2000 à média brasileira de escolaridade vem aumento, trazendo um interesse na ascensão profissional (Pereira et al., 2016).

Ao visualizar a classificação econômica dos agentes de nível médio da enfermagem, segundo o Critério Brasil de Classificação Econômica, que visa identificar o poder de consumo das famílias, a maioria dos profissionais se classificou no nível "B2" (46,69\%), tendo a estimativa média de renda familiar de R \$ 5.641,64. Essa dissonância pode ser vista também em outros estudos, visto que os critérios da ABEP levam em consideração os bens de consumo das famílias, podendo não refletir com precisão o real nível socioeconômico dos indivíduos dos estudos. Vale ainda lembrar de que as diversas condições de vida, por exemplo, uma maior renda e a qualidade de vida, são determinantes no processo de saúde/doença dos indivíduos.

Outro efeito dessa renda familiar, principalmente para o público desta pesquisa, pode ocorrer pelo fato de que a maioria exerceu função em cargo público (71\%), no qual há uma variação importante e não compatível com a carga de trabalho executada pela equipe de enfermagem e normalmente os salários são maiores em relação ao setor privado, com maior estabilidade e menos oscilações das taxas de crescimento econômico (Faria; Machado, 2016; Santos et al., 2017; ABEP, 2019).

No que tange a trajetória profissional dos indivíduos, identificou-se que, a partir dos anos 70, compreendeu-se o período com maior investidura na carreira de trabalho na enfermagem, correspondendo a 36,93\%. Isso pode ser explicado pelos marcos históricos ocorridos no estado de Mato Grosso, fazendo com que o trabalho da enfermagem tenha grande importância para a colonização da região estudada, se fortalecendo assim uma enfermagem mais moderna e cientificada (Flores; Almeida; Junior, 2017).

No momento atual deste estudo, 77,73\% dos profissionais se apresentam na condição de aposentados. Quanto aos indivíduos que se encontravam aposentados, 43,10\% declararam que recebem o benefício há mais de 10 anos. Logo, o motivo mais apontado para a aposentadoria foi tempo de serviço, com 60,80\%.

Destarte, vale ressaltar que dentre os que se encontravam em atividade, uma parcela destes $(72,73 \%)$, estavam na situação de aposentados, mas em atividade. Isso se dá, segundo autores, pelas necessidades materiais, psicológicas e sociais dos indivíduos, difundindo o sentimento de ainda poder contribuir com a profissão, de se autoconhecer e buscar um melhor rendimento e qualidade de vida (Hyeda; Costa, 2017).

Ao averiguar as características de trabalho, em relação aos vínculos empregatícios dos participantes, o maior contrato de permanência desses profissionais foi em órgãos públicos, com 71,10\%. Ao apresentar a carga horária em consonância com a natureza dos diferentes vínculos empregatícios, a jornada de trabalho que mais se destacou foi a de 40 horas semanais, realidade esta que ainda permeia na atuação dos profissionais de enfermagem como apontado em estudos realizados recentemente, como os de Alves, Guirardello (2016), Fernandes, Nitsche, Godoy (2017) e Meireles et al., (2018).

Outra característica evidenciada foi a de predominância da área de atuação hospitalar entre os indivíduos, com 72,50\%. O que foi evidenciado também por Machado et al., (2016d), em que a área hospitalar compreende a modalidade institucional em que os profissionais de enfermagem mais desenvolvem suas atividades (56\%).

A respeito da atuação dos profissionais em mais de um vínculo empregatício, ressalta-se que apenas 43,90\% informaram possuir multiemprego em algum momento da trajetória de seu trabalho, tendo estes mencionado ter mantido de 5 a 10 anos de duplo vínculo trabalhista.

De acordo com Souza et al., (2017), o trabalho na enfermagem quando executado em escalas de plantão, propicia a este profissional a possibilidade de manter mais de um emprego. Todavia, este acúmulo laboral acarreta ao profissional 
diversos problemas, que acabam interferindo diretamente no trabalho e em sua qualidade de vida, principalmente para os profissionais que estão acima dos 60 anos de idade (Cantele et al., 2017; Silva et al., 2018).

Nesse contexto, o enfermeiro necessita, além de prestar o cuidado, ter o autocuidado, estar bem consigo para prestar uma assistência de qualidade, tendo um planejamento sobre seu envelhecimento, pois com o aumento da expectativa de vida, há também um aumento no tempo de trabalho de cada um, causando desgastes ao profissional, acelerando, com isso, seu processo de envelhecimento (Costa; Fortes; Soane, 2019).

\subsection{Hábitos de vida e perfil de saúde dos agentes de nível médio da enfermagem}

Dentre os participantes da pesquisa, apenas $4,50 \%$ se autodeclararam tabagistas. Dado que vai de encontro com estudos recentes com a população de enfermagem no Brasil, como os Santos et al., (2017). Contudo, em outros países, como nos estudos de Chandrakumar, Adams (2015) e Nichter et al., (2018), a prevalência do tabaco aumentou significativamente, principalmente entre os profissionais de enfermagem.

Em relação ao uso de bebida alcoólica, 17,10\% relataram consumo em suma apenas em finais de semana. Entretanto, apesar do baixo quantitativo, estudos como os de Rocha, David (2015) e Scholze et al., (2017), abordam que o consumo de bebidas alcoólicas tem tido aumento significativo na população e igualmente entre os profissionais de enfermagem, tornando este um problema de saúde pública.

Já ao averiguar o Índice de Massa Corpórea (IMC), destacaram-se as prevalências nos parâmetros de sobrepeso e obesidade, correspondendo a 40,07\% e 30,66\% respectivamente. Ao contrastar o resultado acima com o de prática de atividade física, observou-se que apenas 40,10\% alegaram realizar alguma atividade durante a semana. Com relação a prática de atividades de lazer, obtivemos os mais diversos relatos, por exemplo, caminhar, ir à igreja, ir para o sítio, dançar, viajar, dentre outras, que categorizamos como "outros", o que não era compatível com as sugestões dadas no instrumento.

$\mathrm{Na}$ enfermagem, um estudo evidenciou que os técnicos e auxiliares de enfermagem apresentaram menores valores de nível de atividade física quando comparados com profissionais de nível superior, uma vez que o ambiente insalubre e penoso, alta carga horária de trabalho, baixa remuneração e multiemprego impossibilitam a realização de atividade por estes profissionais (Barbosa et al., 2018).

Quanto às condições de saúde, 33,80\% informaram possuir alguma doença ocupacional diagnosticada. Entre aqueles que ainda continuavam em atividade profissional na enfermagem $(n=55), 36,36 \%$ estiveram afastados do trabalho no último ano por motivo de saúde. A maioria relatou não apresentar nenhuma limitação física. Sobre possuir familiar dependente de seus cuidados em detrimento de algum agravo à saúde, 19,50\% alegaram possuir essa incumbência.

Resultado este que contraria outros estudos, como o de Santos et al., (2017) e Marques et al., (2015), no qual 65\% e $59 \%$ respectivamente dos profissionais de enfermagem relataram afastamentos por motivos de saúde. Com relação a este dado, estudos demonstram que o maior índice de afastamento do trabalho (81\%), é entre os técnicos e auxiliares de enfermagem.

Dado este preocupante, uma vez que a maioria do contingenciamento da enfermagem é composta por esses profissionais, que, em seu exercício diário, são responsáveis por várias atividades, principalmente as do ponto de vista ergonômico, e com o agravante de estar na terceira idade, vem a refletir em altas taxas de afastamentos, licenças e aposentadorias por invalidez (Santos et al., 2017).

Considerando as cargas sociais e de trabalho dos profissionais de enfermagem, além dos diversos fatores específicos da profissão, como estresse, rotatividade de turnos, jornada noturna, multiemprego e sobrecarga de trabalho, somadas aos hábitos de vida impróprios, como inatividade física, tabagismo, álcool, obesidade, estas podem vir a refletir na saúde dos agentes de nível médio da enfermagem, visto que a maioria desses profissionais $(74,20 \%)$, citaram ter uma ou mais Doenças 
Crônicas Não Transmissíveis - DCNT, sendo as de maior prevalência as doenças cardiovasculares, com 62,02\%, seguidas das doenças endócrinas, com 26,48\% dos casos (Tabela 1).

Tabela 1 - Prevalência de doenças crônicas entre os 287 indivíduos entrevistados. Cuiabá, Mato Grosso (2019).

\begin{tabular}{lllll}
\hline Variáveis & & N & & \% \\
\hline Doenças crônicas & Sim & Não & Sim & Não \\
\hline Cardiovascular & 178 & 109 & 62,02 & 37,98 \\
Endócrino & 76 & 211 & 26,48 & 73,52 \\
Musculoesquelético & 33 & 254 & 11,50 & 88,50 \\
Pulmonar & 5 & 282 & 1,74 & 98,26 \\
Visual/auditiva & 4 & 283 & 1,39 & 98,61 \\
Imunológica & 4 & 283 & 1,39 & 98,61 \\
Hepática & 2 & 285 & 0,70 & 99,30 \\
Psiquiátrica & 1 & 286 & 0,35 & 99,65 \\
Sistema nervoso central & 1 & 286 & 0,35 & 99,65 \\
\hline
\end{tabular}

Fonte: Dados da pesquisa. Autores.

As altas prevalências de DCNT têm sido observadas na enfermagem (Assunção, 2016; Domingues et al., 2019), e estudos têm evidenciado que o estresse é um dos principais fatores de risco para o desenvolvimento de DCNT, principalmente as de cunho cardiovascular, que neste estudo teve maior prevalência, pois as condições de trabalho e sociais às quais estes profissionais estão submetidos contribuem para o aumento da sua vulnerabilidade (Magalhães et al., 2014; Kotekewis et al., 2017).

Quanto às doenças do sistema endócrino, temos a diabetes mellitus, responsável por 26,48\% dos resultados. Na enfermagem, o diabetes mellitus ocorre muitas vezes pelo estilo de vida destes profissionais. Outros fatores como alteração no padrão de sono, hábitos alimentares, a alta taxa de sobrepeso e obesidade e a inatividade física são fatores que contribuem para o risco de adquirir a doença (Brasil, 2013; Domingues et al., 2019).

Sobre os distúrbios musculoesqueléticos estes também são apontados por uma parcela dos participantes deste estudo $(11,50 \%)$. O que vai de encontro com o resultado acerca da relação às limitações físicas decorrentes do trabalho, onde $18 \%$ declararam possuir alguma limitação e, destes, grande parcela (73\%), autodeclararam possuir limitações relativas a articulações, tornozelo, joelho e ombro, problemas de coluna e incapacidade de pegar peso, resultados que vão de encontro com outros estudos realizados com os profissionais de enfermagem (Santos et al., 2017; Souza et al., 2018a).

Essas alterações musculoesqueléticas ocorrem devido às caraterísticas do trabalho da enfermagem, principalmente em relação ao profissional de nível médio, uma vez que estes realizam as tarefas que exigem maior esforço físico, além de exercerem uma assistência direta ao paciente (Pacheco et al., 2016).

Freire e Santiago (2017), relatam que muitos trabalhadores da enfermagem não percebem o risco ao qual estão expostos, diante disso e em referência às implicações das DCNT, cerca de $74 \%$ dos participantes relataram fazer uso de um ou mais medicamento de uso contínuo, tendo as classes medicamentosas com maiores frequências apresentadas os antihipertensivos $(67,72 \%)$, hipoglicemiantes orais/insulina $(23,69 \%)$, vitaminas ou suplementos $(5,92 \%)$. Embora tenha apenas um relato de doença psiquiátrica, houve a presença de uso de psicoativos nos relatos dos indivíduos entrevistados (Tabela 2). 
Tabela 2 - Utilização de medicação contínua entre os 287 indivíduos entrevistados. Cuiabá, Mato Grosso (2019).

\begin{tabular}{lllll}
\hline Variáveis & \multicolumn{2}{c}{$\mathbf{N}$} & & \% \\
\hline Fármacos & Sim & Não & Sim & Não \\
\hline Anti-hipertensivo & 180 & 107 & 62,72 & 37,28 \\
Hipoglicemiantes orais/insulina & 68 & 219 & 23,69 & 76,31 \\
Vitaminas ou suplementos & 17 & 270 & 5,92 & 94,08 \\
Psiquiátricos & 14 & 273 & 4,88 & 95,12 \\
Hormonais & 12 & 275 & 4,18 & 95,82 \\
Antirreumáticos & 7 & 280 & 2,44 & 97,56 \\
Hipolipemiantes & 6 & 281 & 2,09 & 97,91 \\
Anticoagulantes & 3 & 284 & 1,05 & 98,95 \\
Antiplaquetário & 2 & 285 & 0,70 & 99,30 \\
Diurético & 1 & 286 & 0,35 & 99,65 \\
\hline
\end{tabular}

Fonte: Dados da pesquisa. Autores.

Quanto ao uso de medicamentos de forma contínua, observa-se concordância nos resultados deste estudo quando se compara com diversos estudos sobre os medicamentos mais utilizados na população idosa e, ainda, dentre os medicamentos que mais são utilizados pelos profissionais de enfermagem (Souza et al., 2012; Domingues et al., 2019).

Apesar de ter sido observado em aproximadamente 5\% dos participantes o uso de medicamento de ordem psíquica, este se sobressai em relação ao número de profissionais que relataram doenças dessa categoria (0,35\%). Pereira et al., (2016), discorrem que o uso dessas substâncias ocasiona transformações na mente, corpo e na conduta dos profissionais, trazendo riscos tanto para quem consome quanto para quem convive com eles.

\subsection{Qualidade de vida relacionada à saúde dos agentes de nível médio da enfermagem}

Ao analisar os dados relativos à Qualidade de Vida Relacionada à Saúde - QVRS, dos participantes (Tabela 3), a média obtida pelos escores dos domínios foi de 69,69 pontos, escores representativos de uma boa qualidade de vida relacionada à saúde. Esses dados são semelhantes aos encontrados em estudos recentes com a população de enfermagem (Souza et al., 2018b; Rocha; Ruiz, 2019).

Entretanto, ao verificar os domínios separadamente, constatou-se que o domínio com melhor média foi o de aspectos sociais $(83,29 \%)$ e o com menor média foi o de estado geral da saúde $(60,66 \%)$ (Tabela 3$)$.

Tabela 3 - Média, Mediana e Desvio padrão por domínios da qualidade de vida relacionada à saúde dos 287 indivíduos entrevistados. Cuiabá, Mato Grosso (2019).

\begin{tabular}{llcc}
\hline Variáveis & Média & Mediana & Desvio padrão \\
\hline Aspectos sociais & 83,29 & 100,00 & 22,15 \\
Saúde mental & 74,33 & 76,00 & 17,41 \\
Limitação por aspectos emocionais & 72,71 & 100,00 & 54,33 \\
Capacidade funcional & 71,03 & 75,00 & 25,62 \\
Qualidade de Vida & 69,69 & 72,50 & 16,40 \\
Vitalidade & 69,80 & 70,00 & 17,39 \\
Limitação por aspectos físicos & 67,95 & 75,00 & 45,89 \\
Dor & 61,48 & 61,00 & 26,43 \\
Estado geral da saúde & 60,66 & 62,00 & 18,34 \\
\end{tabular}


O domínio de aspectos sociais foi o que mais se destacou em relação aos demais, já no estudo de Costa et al., (2017), foram encontradas médias para o domínio aspectos sociais de 22,5, dado contrário ao encontrado nesta pesquisa. Outro estudo sobre a qualidade de vida em trabalhadores da área da saúde, estando ou não em atividade, demonstra que o escore obtido no domínio aspectos sociais em trabalhadores da saúde ativos (84), foi maior do que nos que se encontravam em afastamento (56) (Dutra; Costa; Sampaio, 2016).

Já o domínio estado geral da saúde foi o mais afetado entre os participantes deste estudo, conforme visualizado também por Costa et al., (2017), no qual a média do escore do domínio foi de 25. Isso pode ser levado em consideração em consonância com o trabalho realizado pelos profissionais do estudo, principalmente ao chegar à terceira idade, em que já se passa por alterações fisiológicas e emocionais que são características dessa fase. Problemas estes que contribuem no estado geral de saúde destes profissionais.

Os demais domínios deste estudo, como capacidade física, limitação por aspectos físicos, dor, vitalidade, limitação por aspectos emocionais e saúde mental, apresentaram médias superiores a 60 pontos. Logo, podem ser consideradas como tendo uma boa pontuação sobre a avaliação da qualidade de vida em relação a esses domínios.

Costa et al., (2017), procuraram avaliar a qualidade de vida de profissionais da enfermagem que atuavam em ambiente hospitalar e encontraram médias praticamente iguais as deste estudo, superiores a 60 pontos, exceto nos domínios de Dor (22,4), Estado Geral da Saúde (25) e Aspectos Sociais (22,5). Já no estudo de Silva e Guimarães (2016a), identificou-se que os técnicos e auxiliares de enfermagem apresentaram índices de qualidade de vida maiores do que os enfermeiros, exceto no domínio relacionado à capacidade funcional.

Quanto à idade, os 8 domínios do SF-36 apresentaram redução na pontuação dos escores médios com o aumento da idade, sendo 90 pontos compreendendo a faixa etária de 20 a 30 anos, e diminuição para 69,09 para profissionais com idade acima dos 60 anos.

\subsection{Diferenciação estatística entre as variáveis dependente e independente pelo teste de Kruskal-Wallis}

O teste estatístico de Kruskal-Wallis foi aplicado em todas as variáveis independentes do estudo, porém só foram destacadas aquelas que apresentaram valor estatístico significativo, quando comparado com a variável dependente do estudo.

Os indivíduos que declararam não ter filhos apresentaram mediana estatisticamente significativa para o domínio dor $(p=0,004)$ e QVRS ( $p=0,042)$. Com relação a este achado, temos dados do estudo de Silva e Guimarães (2016a), que ao analisar a variável filhos, identificaram alterações em todos os domínios do SF-36, sendo o domínio mais afetado o de capacidade funcional, com associação estatística significante ( $\mathrm{p}=0,015)$.

Entre os indivíduos que eram aposentados, estes apresentaram mediana inferior e diferenciação estatística quando comparados aos que permaneciam em atividade para os domínios capacidade funcional $(p=0,001)$, limitação por aspectos físicos ( $p=0,001)$, dor ( $\mathrm{p}=0,024)$, saúde mental $(\mathrm{p}=0,001)$ e na QVRS ( $\mathrm{p}=0,001)$.

Com relação a este achado, podemos citar dados de Dutra, Costa e Sampaio (2016), que apontaram uma associação entre estar trabalhando e uma qualidade de vida, na qual em todos os domínios os indivíduos que estavam em atividade apresentaram escores melhores do que aqueles se encontravam afastados do trabalho. O que vai de encontro com resultados do estudo de Lima et al., (2015), onde referem que os indivíduos ativos apresentaram melhores escores nos domínios aspectos físicos, aspectos sociais e saúde mental em relação aos que estavam em inatividade, corroborando com os resultados deste estudo.

A possibilidade de comparar e avaliar a qualidade de vida de trabalhadores ativos e que estão fora do mercado de trabalho demonstra o quanto a participação em atividades laborais influência de maneira positiva a percepção da saúde e a qualidade de vida destes trabalhadores. 
Com relação à variável carga horária mais praticada, os indivíduos que trabalhavam 30 horas semanais apresentaram mediana estatisticamente significativa em relação aos que trabalhavam 44 horas semanais para os domínios capacidade funcional $(\mathrm{p}=0,045)$ e dor $(\mathrm{p}=0,011)$. Diante este achado, foi evidenciado que os agentes de nível médio da enfermagem na terceira idade apresentaram melhor qualidade de vida nos domínios capacidade funcional e dor com relação à carga horária cumprida.

Corrobora com nossos resultados estudo de Silva e Guimarães (2016a), onde discorrem que a carga horária de trabalho é um fator condicionante para a qualidade de vida dos profissionais de enfermagem, e jornadas menores que 40 horas semanais demonstram melhoria na qualidade de vida.

A respeito da variável área de atuação, a mesma foi recategorizada em duas categorias para a análise, tendo os indivíduos que praticaram assistência direta e os de assistência indireta. Verificou-se que os profissionais que realizam assistência indireta apresentaram medianas melhores de qualidade de vida para o domínio estado geral da saúde $(p=0,009)$, em relação a àqueles que prestam assistência direta.

Costa et al., (2017), em seu estudo sobre qualidade de vida, descrevem que o domínio estado geral da saúde foi um dos domínios mais afetados no seu estudo, o que vai de encontro com resultados de Fraga, Calvetti e Lazzarotto (2019), que descreveram que os técnicos de enfermagem apresentaram escores mais comprometidos em relação aos enfermeiros, a rotina diária que estão expostos que contribuem diretamente no comprometimento do estado geral da saúde destes profissionais.

Quanto aos indivíduos que relataram possuir mais de um vínculo, as medianas foram estatisticamente significativas para os domínios aspectos sociais ( $\mathrm{p}=0,002)$, saúde mental $(\mathrm{p}=0,013)$ e QVRS $(\mathrm{p}=0,040)$ em relação aos que negaram tal prática. Contrariando esse resultado, Souza et al., (2018b), abordam que manter mais de um vínculo resulta em grandes desgastes físicos e mentais, aumentando, assim, o nível de estresse e falhas durante a execução do trabalho, refletindo no cuidado prestado e trazendo reflexos negativos à qualidade de vida.

Ainda no que tange a jornada dupla de trabalho o estudo de Albuquerque, Silva e Oliveira (2018), descrevem que profissionais que possuem vários vínculos apresentaram em aspectos sociais $(67,2)$, saúde mental $(64,9)$, vitalidade $(57,1)$ e dor $(62,5)$ uma qualidade de vida menos favorecida em relação aos profissionais que possuem apenas um vínculo. No entanto, vale destacar que não houve diferença significativa entre nenhum domínio do SF-36 quanto à jornada de trabalho, entre quem realizava dupla jornada e quem realizava apenas uma, pois os domínios do estudo apresentaram escores acima de 50 pontos, o que leva, consideravelmente, a uma boa qualidade de vida.

Com base no consumo de álcool os indivíduos que autodeclaram consumir bebida alcoólica, apresentaram medianas superiores e estatisticamente significativas em relação aos que negaram consumir álcool, nos domínios capacidade funcional ( $p$ $=0,012)$, limitação por aspectos físicos $(\mathrm{p}=0,001)$, dor $(\mathrm{p}=0,044)$, estado geral da saúde $(\mathrm{p}=0,009)$ e QVRS $(\mathrm{p}=0,001)$.

Estudo de Junqueira et al., (2017), identificaram predominância de comportamentos de saúde mais saudáveis em relação ao consumo de álcool entre os técnicos de enfermagem. Esse dado condiz com o resultado apresentado neste estudo, no qual 17,10\% relataram consumo de álcool e, dentre estes, 71,40\% mencionaram fazer o consumo apenas em finais de semana.

Entre os indivíduos que declararam possuir alguma doença crônica, evidenciou-se uma menor mediana com estatística significativa nos domínios capacidade funcional $(\mathrm{p}=0,042)$, dor $(\mathrm{p}=0,001)$, estado geral da saúde $(\mathrm{p}=0,001)$ e QVRS $(\mathrm{p}=$ 0,006), em relação àqueles que não declararam possuir doença crônica. Tendo ainda os que informaram possuir alguma doença ocupacional, constatou-se também menor mediana com estatística significativa para os domínios capacidade funcional $(\mathrm{p}=$ $0,001)$, limitação por aspectos físicos ( $p=0,017)$, dor $(p=0,002)$ e QVRS $(p=0,015)$.

Mendonça et al., (2015), realizaram em seu estudo, a avaliação da qualidade de vida de mulheres e relacionou com as doenças crônicas não transmissíveis. No estudo mais da metade dos participantes relataram apresentar alguma doença crônica, tendo as mais visualizadas as doenças osteomioarticulares, hipertensão arterial e diabetes mellitus, além de que uma minoria 
apresentou associação para mais de duas doenças. Resultado este semelhante ao deste estudo, no qual a maioria dos participantes era do sexo feminino e uma grande parcela (74\%), declararam apresentar alguma doença crônica, sendo as mais presentes doenças do sistema cardiovascular, endócrino e musculoesquelético.

No que se refere aos resultados relativos às doenças ocupacionais, os indivíduos que não apresentaram doenças ocupacionais apresentaram melhores medianas e significância estatística em detrimento daqueles que referiam obter doença ocupacional, nos domínios capacidade funcional, limitação por aspectos físicos, dor e qualidade de vida relacionada a saúde.

Estudos vêm comprovando os baixos índices de condições de trabalho, com sintomas físicos, emocionais e saúde em geral. Quanto aos profissionais de enfermagem, esses fatores estão diretamente relacionados ao contexto ocupacional no trabalho (Costa et al., 2017), que ocasionam ao trabalhador uma carga mental que interfere diretamente na qualidade de vida e no trabalho (Zavala et al., (2016).

Em meio aos indivíduos que relataram algum tipo de limitação física, eles apresentaram medianas inferiores com estatística significativa nos domínios limitação por aspectos físicos ( $p=0,004)$, dor ( $(p=0,012)$ e QVRS ( $p=0,007)$.

Hyeda e Costa (2017), em seu estudo, descreveram que as características do trabalho da enfermagem, as condições de trabalho e os riscos ergonômicos gerados durante as atividades laborais podem vir a acometer a saúde do trabalhador, fazendo com que este apresente limitações ou até mesmo incapacidade relativas ao trabalho. O estudo acima entra em similaridade com os resultados deste estudo, visto que doenças musculoesqueléticas apresentaram maior frequência na população dos agentes de nível médio da enfermagem na terceira idade, pois os trabalhadores de enfermagem, especialmente os de nível médio, realizam os cuidados mais diretos ao paciente, o que leva a maiores riscos de agravo à saúde.

Por fim, quanto a prática de atividade física, aqueles que relataram praticar, constataram-se maiores medianas em quase todos os domínios, sendo eles: capacidade funcional $(\mathrm{p}=0,006)$, dor $(\mathrm{p}=0,048)$, estado geral da saúde $(\mathrm{p}=0,043)$, aspectos sociais $(\mathrm{p}=0,007)$, saúde mental $(\mathrm{p}=0,001)$ e QVRS $(\mathrm{p}=0,002)$, em relação àqueles que relataram não praticar atividade física.

Com relação ao resultado deste estudo, achados de Acioli et al., (2013) e Souza et al., (2018b), descrevem que os profissionais de saúde fisicamente ativos apresentaram melhores avaliação relacionadas à saúde nos domínios capacidade funcional, vitalidade e saúde mental, o que pode ser utilizado como uma ferramenta para os gestores da área da saúde a fim de avaliar e buscar uma melhor qualidade de vida relacionada à saúde a este público. Dados estes que vão de encontro com nossos achados, tendo influenciado significativamente em quem pratica atividade física, levando a um aumento nos escores de todos os domínios do SF-36.

\section{Conclusão}

O estudo permitiu evidenciar que no que tange à qualidade de vida relacionada à saúde dos agentes de nível médio da enfermagem utilizando como medida o instrumento SF-36, encontrou-se que a pontuação média dos profissionais foi cerca de 69. Esse escore se caracteriza como uma pontuação considerada de boa qualidade de vida, porém o fato de os profissionais estarem na terceira idade e aposentados pode ter contribuído para esse resultado em razão das características que esses indivíduos apresentavam, muitas vezes com doenças e limitações físicas ou com problemas familiares, acarretando diversos abalos emocionais.

Além disso, ao analisar os domínios individualmente, constatou-se que o domínio com resultados mais favoráveis para uma considerada boa qualidade de vida relacionada à saúde foi o de aspectos sociais devido aos participantes estarem conscientes sobre sua situação de saúde, tanto física como emocional, e as interações com amigos, colega de trabalho e principalmente a família. 
O domínio com menor escore foi o de estado geral da saúde, que está ligado mais às percepções dos profissionais sobre a sua saúde, sendo mais subjetivo em relação aos outros domínios. Como discutido no estudo, diversas literaturas vêm demonstrando que os domínios do instrumento SF-36 exibiram escores inferiores aos encontrado neste estudo. Entretanto, a maioria das pesquisas foi realizada com profissionais que se encontravam em atividade, em diversos setores de atuação na enfermagem, contendo diversas idades.

Neste contexto este estudo é de relevância, a se considerar que se idealizou que os achados aqui apresentados possam contribuir para melhorias na atuação destes profissionais e para os avanços em relação à saúde do trabalhador, ressaltando a importância para a categoria da aprovação de projetos de lei que visem proporcionar melhores condições de trabalho. Além de fornecer base para exploração da saúde e envelhecimento em trabalhadores da categoria, contribuindo para ampliação e maiores possibilidades que envolvem o tema enfermagem e envelhecimento, na intencionalidade de provocar aos interessados uma preparação para essa fase.

Por fim, considera-se que não há pretensão de se esgotar o assunto nesta pesquisa, e sim servir de auxílio a futuras pesquisas que acendem ao tema, e auxiliando aos demais, espera-se que o estudo possa contribuir para pensar estratégias de fortalecimento a saúde do trabalhador para que a classe obtenha uma melhor qualidade de vida relacionada à saúde.

\section{Referências}

ABEP. (2021). Critérios de Classificação Econômica Brasil. <http://www.abep.org/criterio-brasil〉.

Andrade, M. M. (2002). Como preparar trabalhos para cursos de pós-graduação: noções práticas. (5ª ed.) São Paulo: Atlas.

Neto, A. A., Araújo, R., Pitangui, A., Menezes, L., França, E., Costa, E., Andrade, F., \& Junior, M. C. (2013). Qualidade de vida e nível de atividade física de profissionais de saúde de unidades de terapia intensiva. Revista Brasileira Atividade Física \& Saúde. 18(6), 711-719. https://doi.org/10.12820/rbafs.v.18n6p711.

Alves, D. F. S., \& Guirardello, E. B. (2016). Ambiente de trabalho da enfermagem, segurança do paciente e qualidade do cuidado em hospital pediátrico. Revista Gaúcha Enfermagem. 37(2), e58817.

Amaral, J. F., Ribeiro, J. P., \& Paixão, D. X. (2015). Qualidade de vida no trabalho dos profissionais de enfermagem em ambiente hospitalar: uma revisão integrativa. Revista Espaço para a Saúde. 6(1), 66-74.

Barbosa, T. C., Mota. A. N., Mendonça, G. N., \& Quaresma, F. R. P. (2018). Nível de atividade física de profissionais de duas unidades de pronto atendimento na região norte do Brasil. Revista Brasileira de Fisiologia do Exercício. 17(2),104-112. https://doi.org/10.33233/rbfe.v17i2.2266.

Brasil. (2013). Estratégias para o cuidado da pessoa com doença crônica: diabetes mellitus. Cadernos de Atenção Básica, n. 36.

Brasil. (1986). Lei 7.498, de 25 de junho de 1986. Dispõe sobre a Regulamentação do Exercício da Enfermagem e dá outras providências. Brasília (DF): Ministério da Saúde.

Brasil. (1994). Lei 8.967, de 25 de dezembro de 1994. Altera a redação do parágrafo único do art. 23 da Lei nº 7.498 , de 25 de junho de 1986, que dispõe sobre a regulamentação do exercício da enfermagem e dá outras providências. Brasília (DF): Ministério da Saúde.

Brasil. (2017). Resolução $N^{\circ} 466$, de 12 de dezembro de 2012. <http://www. conselho.saude.gov.br/web_comissoes/conep/index.html>.

Brasil. (2016). Resolução $N^{o} \quad 510, \quad$ de $07 \quad$ de $\quad$ abril $\quad$ de $\quad$ http://conselho.saude .gov.br/resolucoes/2016/reso510.pdf>.

Cantele, A. B., Araújo, B.N., Pellizzari, G., \& Bidel, R. M. R. (2017). Envelhecimento ativo: o conhecimento dos técnicos de enfermagem da estratégia saúde da família. Revista Saúde e Desenvolvimento. 11(9), 156-171.

Chandrakumar, S., \& Adams, J. (2015). Attitudes to smoking and smoking cessation among nurses. Nursing Standard (2014+). 30(9), 36.

Costa, A. C., Fortes, A. F. A., \& Soane, A. M. N. C. (2019). A imagem e o preparo do enfermeiro acerca do próprio envelhecimento. Enfermagem Brasil. 12(4), 198-205.

Costa, K. N. F. M., et al. (2017). Qualidade de vida relacionada à saúde dos profissionais de enfermagem. Revista enfermagem UFPE on line, p. 881-889.

Domingues, J. G., Silva, B. B. C., Bierhals, I. O., \& Barros, F. C. (2019). Doenças crônicas não transmissíveis em profissionais de enfermagem de um hospital filantrópico no Sul do Brasil. Epidemiologia e Serviços de Saúde. 28(2), e2018298. 10.5123/S1679-49742019000200011.

Dutra, F. C. M. S., Costa, L. C., \& Sampaio, R. F. (2016). A influência do afastamento do trabalho na percepção de saúde e qualidade de vida de indivíduos adultos. Fisioterapia e Pesquisa. 23(1), 98-104. 
Faria, E. B. L., \& Machado, C. J. (2016). Qualificação para o Trabalho em Saúde: Um Estudo Exploratório sobre Escolaridade e Cargo na Secretaria Municipal de Saúde de Belo Horizonte, 2009. Revista Brasileira de Educação e Cultura| RBEC. 14, 34-60.

Fernandes, L. S., Nitsche, M. J. T., \& Godoy, I. (2017). Bornout Syndrome in nursing professionals from an Intensive Care Unit. Revista Fundação Care Online. 9(2), 551-557.

Flores, C. A. S., Almeida, P. B. A., \& Junior, E. M. J. (2017). Investigação e Documentação Histórica da Enfermagem na Região Norte do Estado de Mato Grosso - Brasil. História da Enfermagem Revista eletrônica [Internet].8(1), 18-26.

Freire, A. K. S., \& Santiago, E. J. P. (2017). Doenças ocupacionais nos trabalhadores de enfermagem e educação em saúde: revisão integrativa. Revista Saúde e Desenvolvimento. 11(6), 202-218.

Freire, M. N., \& Costa, E. R. (2016). Qualidade de vida dos Profissionais de Enfermagem no Ambiente de Trabalho. Revista Enfermagem Contemporânea. 5(1):151-158. http://dx.doi.org/10.17267/2317-3378rec.v5i1.871.

Gil, A. C. (2010). Métodos e técnicas de pesquisa social. In: Métodos e técnicas de pesquisa social. Atlas.

Hyeda, A., \& Costa, É. S. M. (2017). A relação entre a ergonomia e as doenças crônicas não transmissíveis e seus fatores de risco. Revista brasileira medicina do Trabalho. 15(2): 173-181.

Junqueira, M. A. B., Ferreira, M. C. M., Soares, G. T., Brito, I. E., Pires, P. L. S., Santos, M. A., \& Pillon, S. C. (2017). Uso de álcool e comportamento de saúde entre profissionais da enfermagem. Revista da Escola de Enfermagem da USP. 51, e03265. 10.1590/S1980-220X2016046103265.

Kotekewis, K., Ribeiro, R. P., Ribeiro, B. G. A., \& Martins, J. T. (2017). Doenças crônicas não transmissíveis e o estresse dos trabalhadores de enfermagem de bloco cirúrgico. Enfermería Global. 16(2), 295-314. 10.6018/eglobal.16.2.252581.

Lima, L. M., Souza, R. J. S., Cunha, M. R. H., Leopoldo, A. S., \& Lima-Leopoldo, A. P. (2017). Prevalência de Sobrepeso e Obesidade em Idosas do Centro de Convivência para a Terceira Idade de Vitória. ES. Revista Brasileira Ciência da Saúde. 21(2), 119-26.

Machado, M. H., Wermelinger, M., Vieira, M., Oliveira, E., Lemos, W., Aguiar Filho, W., Lacerda, A. F., Santos, M. R., Souza Junior, P. B., Justino, E, \& Barbosa, C. (2016a). Características gerais da enfermagem: o perfil sócio demográfico. Enfermagem em Foco.7( 1/4), 11-17.

Machado, M. H., Wermelinger, M., Vieira, M., Oliveira, E., Lemos, W., Aguiar Filho, W., Lacerda, A. F., Santos, M. R., Souza Junior, P. B., Justino, E, \& Barbosa, C. (2016b). Aspectos gerais da formação da enfermagem: o perfil da formação dos enfermeiros, técnicos e auxiliares. Enfermagem em Foco. 7( ESP), 15-34.

Machado, M. H., Wermelinger, M., Vieira, M., Oliveira, E., Lemos, W., Aguiar Filho, W., Lacerda, A. F., Santos, M. R., Souza Junior, P. B., Justino, E, \& Barbosa, C. (2016c). Condições de trabalho da enfermagem. Enfermagem em Foco. 6(1/4), 79-90.

Machado, M. H., Wermelinger, M., Vieira, M., Oliveira, E., Lemos, W., Aguiar Filho, W., Lacerda, A. F., Santos, M. R., Souza Junior, P. B., Justino, E, \& Barbosa, C. (2016d). Mercado de trabalho da enfermagem: aspectos gerais. Enfermagem em Foco. 7 (ESP), 35-53.

Magalhães, F. J., Mendonça, L. B. A., Rebouças, C. B. A., Lima, F. E. T., Custódio, I. L., \& Oliveira, S. C. (2014). Fatores de risco para doenças cardiovasculares em profissionais de enfermagem: estratégias de promoção da saúde. Revista Brasileira de Enfermagem. 67 (3), $394-400$.

Marques, D. O., Pereira, M. S., Silva e Souza, A. C., Vila, V. S. C., Almeida, C. C. O. F., \& Oliveira, E. C. (2015). O absenteísmo - doença da equipe de enfermagem de um hospital universitário. Revista Brasileira de Enfermagem. 68(5), 876-82. 10.1590/0034-7167.2015680516i.

Medronho, R. A. (2009). Epidemiologia. São Paulo: Atheneu.

Meira, E. D., Reis, L. A., Gonçaleves, L. H. T., Rodrigues, V. P., \& Philipp, R. R. (2017). Vivências de mulheres cuidadoras de pessoas idosas dependentes: orientação de gênero para o cuidado. Escola Anna Nery. 21(2), e20170046. 10.5935/1414-8145.20170046.

Meireles, A. R., Machado, M. G., Silva, M. S., Santos, O. P., Moraes Filho, I. M., \& Santos e Silva Ribeiro, F. M. (2018). Estresse ocupacional da equipe de enfermagem de um serviço de atendimento móvel de urgência. Revista de Divulgação Científica Sena Aires. 7(3), $34-228$.

Mendes, M. A. (2016). Mulheres Chefes de Família: a complexidade e ambigüidade da questão. Anais, p. 1-13.

Mendonça, G. O., Toreti, J. A., Moreira, L. B., Marino, D. N., \& Souza, L. (2015). Relação entre doenças crônicas não transmissíveis e qualidade de vida em mulheres. Arquivo Ciências da Saúde. 22(4), 82 - 86. 10.17696/2318-3691.22.4.2015.312.

Moreira, L. C., \& Ramos, F. R. S. (2004). O processo histórico de trabalho de enfermagem no município de Cuiabá - Mato Grosso. Revista Brasileira de Enfermagem. 57(6), 764-767.

Nichter, M., Carkoglu, A., Nichter, M., Ozcan, s., \& Uysal, M. A. (2018). Engaging nurses in smoking cessation: Challenges and opportunities in Turkey. Health Policy. 122(2), 192-197. 10.1016/j.healthpol.2017.12.007.

Pacheco, E. S. (2016). Prevalence of musculoskeletal symptoms related to nursing work in the hospital field/Prevalência dos sintomas osteomusculares relacionados ao trabalho de enfermagem no âmbito hospitalar/Prevalencia de síntomas musculoesqueléticos relacionados. Revista de Enfermagem da UFPI. 5(4), 31-37.

Pereira, S. S., Teixeira, C. A. B., Reisdorfer, E., Vieira, M. V., Donato, E. C. S. G., \& Cardoso, L. (2016). A Relação entre Estressores Ocupacionais e Estratégias de Enfrentamento em Profissionais de Nível Técnico de Enfermagem. Texto Contexto - Enfermagem. 25(4), e2920014.

Pires, A. S., et al. (2018). A permanência no mundo do trabalho de profissionais de enfermagem com possibilidade de aposentadoria/The permanence in the world of labor of nursing staff with the possibility of retirement. Ciência, Cuidado e Saúde, 12(2), 338-345. 
Research, Society and Development, v. 10, n. 14, e258101421942, 2021

(CC BY 4.0) | ISSN 2525-3409 | DOI: http://dx.doi.org/10.33448/rsd-v10i14.21942

Ribeiro, I. J. S., Carvalho, P. A. L., Constâncio, T. O. S., Yarid, S. D., \& Vilela, A. B. A. (2018). Estudo bibliométrico sobre teses em enfermagem acerca da qualidade de vida. Revista Saúde Contemporânea. 14(1), 1081-1087.

Ribeiro, A. C., Souza, J. F., \& Silva, J. L. (2014). A precarização do trabalho no sus na perspectiva da enfermagem hospitalar. Cogitare Enfermagem. 19 (3), $569-75$.

Rocha, J. C., \& Ruiz, V. M. (2019). Quality of life and work ability of physicians and nurse workers from hospitals. Braz. J. of Develop. 5(11), $23546-23576$.

Rocha, P., \& David, H. M. S. L. (2015). Padrão de consumo de álcool e outras drogas entre profissionais de saúde. SMAD - Revista Eletrônica Saúde Mental Álcool e Drogas (Edição em Português). 11(1), $42-48$.

Santos, S. V. M., Macedo, F. R. M., Resck, Z. M. R., Sanches, R. S. M Nogueira, D. A., \& Terra, F. S. (2017). Características socioeconômicas, epidemiológicas e laborais de profissionais de enfermagem hospitalar. Revista de Enfermagem do Centro-Oeste Mineiro. 7:e1391. 10.19175/recom.v7i0.1391.

Scholze, A. R., Silva, A. D., Martins, J. T., Dálcol, C., Cremer, E., \& Melo, E. C. (2017). Uso de substancias psicoativas entre los trabajadores de enfermería. Revista Portuguesa de Enfermagem de Saúde Mental. 18, 23-30. 2017. 10.19175/recom.v10i0.3737.

Silva, T. P. D., Araújo, W. N., Stival, M. M., Toleto, A. M., Burke, T. N., \& Carregaro, R. L. (2018). Desconforto musculoesquelético, capacidade de trabalho e fadiga em profissionais da enfermagem que atuam em ambiente hospitalar. Revista da Escola de Enfermagem da USP. 52, e03332. 10.1590/S1980$220 \times 2017022903332$.

Souza, D. O., Gonçalves, F. G. A., Pires, A. S., \& David, H. M. S. L. (2017). DaInfluência do neoliberalismo na organização e processo de trabalho hospitalar de enfermagem. Revista Brasileira de Enfermagem. 70 (5), 961-969. 10.1590/0034-7167-2016-0092.

Souza, L. M., Davila, E. S., Scopel, C. D., \& Barbieri, P. N. A. (2018a). Absenteísmo e sintomas osteomusculares em técnicos e auxiliares de enfermagem de unidades de internação hospitalar. Revista de Enfermagem da UFSM. 8(2), 366-379. 10.5902/2179769228685.

Souza, N. V. D. O., Cunha, L. S., Pires, A. S., Gonçalves, F. G. A., Ribeiro, L. V., Lourenço, S. S., \& Silva, F. (2012). Perfil Socioeconômico e de saúde dos trabalhadores de enfermagem da policlínica Piquet Carneiro. Revista Mineira de Enfermagem.16 (2), 232-240.

Souza, V. S., Silva, D. S., Lima, L. V., Teston, E. F., Benedetti, G. M. S., Costa, M. A. R., \& Mendonça, R. R. (2018b). Qualidade de vida dos profissionais de enfermagem atuantes em setores críticos. Rev Cuid. 9(2), 86-2177. 10.15649/cuidarte.v9i2.506.

Tanferri, E. C., Trevisan-Martins, J., Quina-Galdino, M. J., Perfeito-Ribeiro, R., Fernandez-Lourenço-Haddad, M. C., \& Dalmas, J. C. (2017). Calidad de vida de los auxiliares y técnicos de enfermería jubilados enun hospital universitario. Enfermería Global. 16(47), 302-311. 10.6018/eglobal.16.3.256021.

Wermelinger, M., Machado, M. H., Lobato, M. F., Oliveira, E. S., Moyses, N. N., \& Ferraz, W. (2010). A feminilização do mercado de trabalho em saúde. Divulgação em Saúde para Debate. 45, 54-70.

Zavala, M. O. Q., Klinj, T. P., \& Carrillo, K. L. S. (2016). Qualidade de vida no trabalho do pessoal de enfermagem de instituições públicas de saúde. Revista Latino-Americana de Enfermagem. 24, e2713. 10.1590/1518-8345.1149.2713. 